\title{
Automated Toolkit for Encouraging a Producer to Use Innovative Technologies in Environmentally Oriented Economic Development of Mining Regions
}

\author{
Alexey Medvedev ${ }^{1}$, Ivan Kislyakov ${ }^{1}$, Yevgheniya Prokopenko $^{2, *}$, Maria Semenkina ${ }^{3}$, and \\ Kristina Brester ${ }^{4}$ \\ ${ }^{1}$ Kemerovo Institute (branch) of Plekhanov Russian University of Economics, Kemerovo, Russia \\ ${ }^{2}$ T.F. Gorbachev Kuzbass State Technical University, Kemerovo, Russia \\ ${ }^{3}$ University of Applied Sciences Upper Austria, Hagenberg, Austria Softwarepark 11, 4232 \\ ${ }^{4}$ University of Eastern Finland, Department of Environmental and Biological Sciences, 70700 \\ Kuopio,
}

\begin{abstract}
The automated toolkit for assessing environmental and investment attractiveness of a mining region and the results of its application are discussed in the article. This toolkit includes the optimization mathematical model, the algorithms for the interaction between a regional control center and a producer within the territory, as well as the automated software package for their analysis. The use of the optimization mathematical model makes it possible to take into account the maximum economic potential of a producer, which determines, respectively, a mining region's environment pollution potential. Accounting for environmental risks will allow the control center or other decision makers to identify not only the optimal pattern of eco-economic interaction in the region, but also reflect changes in the environmental and investment climate as a combination of economic potential and involved risks. The model and the algorithms of interaction between a regional control center and a producer, as well as the results of their numerical analysis given in this paper, allow considering this toolkit as an effective decision support tool aimed at improving environmental and investment attractiveness of a mining region by encouraging a producer to use the best available technologies and conserve the natural environment.
\end{abstract}

\section{Introduction}

The current development of the world economy leads to stronger economic and innovative technological competition both between countries and specific regions. World producers try to transfer their fixed capital assets to any part of our planet which can offer them the most favorable production conditions in terms of profits; the economy globalization process is going on with the transfer of polluting enterprises to developing countries. Environmental risks are not always fully taken into account in this process, both for the sake of the of the economic development goals and because of the peculiarities of environmental and 
economic policy of specific regions. At the same time, solving the problems of balanced eco-economic development may lead to the need (imperativeness) for a producer in a coal mining region to develop technological innovations in order to use the best available technologies (BAT) in its production. Thus, the development of a toolkit to encourage a producer along the mentioned state in order to reduce the negative impact on the environment is relevant.

To improve the eco-economic environment of a mining region, it is necessary to use essentially innovative, often interdependent factors of its sustainable development, as well as to develop mechanisms for the integrated assessment of environmental risks taking into account the assessment of both a producer's production potential and public interests $[1,3$, 4, 6-10]. The classical approach to the assessment of environmental risks is based on taking into account the potential damage caused by an environmental risk event and the probability of its materialization, which in most cases is determined by statistical or expert methods. The experience in the field of developing mechanisms for reducing environmental risks proposed in the literature can be divided into the following classes: 1) economic accountability mechanisms (a system of standards, quotas, variances from which leads to the imposition of fines), 2) risk reduction stimulating mechanisms (tax breaks, lending environmental protection measures), 3) risk redistribution mechanisms (state, independent, mutual insurance), 4) reservation mechanisms (provision of material and labor resources, facilities, in the event of emergencies), 5) mechanisms of special funds raising and distribution of and 6) regional program management mechanisms that involve all mechanisms, including, inter alia, mechanisms of public authorities' interest alignment [2].

The purpose of this work is to present a systems approach to assessing environmental and investment attractiveness of a region, including a concept, a mathematical model, and an automated system for the support of management decisions, made both by a regional control center (RCC) and by a producer. Here, the mechanism of eco-economic interaction between a RCC and a producer based on the use of an optimization mathematical model (in which an efficiency criterion for a producer is net present value (NPV), and for the RCC the amount of collected taxes, including environmental charges) is proposed. The model is a two-criterion multiparameter linear optimal control problem, which has efficient solution algorithms [5], as well as the corresponding automated software package for its analysis. On the basis of the model, eco-economic interaction management schemes and a set of algorithms for searching for threshold values of the environmental load rate were developed, which encourage a producer to use BAT, which will reduce the negative environmental impact.

\section{Materials and Methods}

Let's define the concept of environmental and economic attractiveness of a territory as a certain combination of factors that take into account the interaction of its economic potential and environmental risks associated with a territory performance. Assume that an eco-economic policy in a region is developed by a RCC that is interested in the efficient performance of a region as an eco-economic system. Suppose also that it is necessary to organize such an interaction between a RCC and a producer in a region, which will contribute to environmental and economic attractiveness of a region by encouraging a producer to use the best available technologies. Following the specified requirement of a region's development, we will quantify the risks arising from such a development as financial losses due to an incorrectly set or not achieved strategic goal of eco-economic development. Let's consider maximization of tax payments, including environmental charges, objectively determined by economic potential of a producer as a strategic goal for a RCC, and maximization of NPV of the production project or economic operations as a 
strategic goal of a producer, taking into account environmental, production, investment, financial and other restrictions of its operations. To quantify the risk, it is necessary to move from the natural expression of production volumes (determination of polluting emissions) to financial (monetary), for example, to the system's performance restoration cost of when the selected risks materialize. To achieve the goal of the study, we will develop an optimization mathematical model of environmental and economic attractiveness of a region. It is expected that eco-economic policy of a RCC will result in the determination of the optimal distribution of the available potential investments and the output of $n$-types of products produced in a mining region, as well as the environmental load factor, at which the two-criterion problem of meeting a RCC and a producer criteria is solved. Suppose, in addition, the known dependencies of risks $\mathrm{r}_{i}=f_{i}(z)$ of the development of system crisis in the $i$-th direction $i=1, \ldots, m ; m$ - is the number of risk directions) of ensuring environmental, economic, financial and other security types from the cost vector $z \in \mathrm{R}^{l}$ (where $l$ is a number of cost items) to avoid them (eliminate or reduce). Note that mathematical modeling of a producer's activities traditionally takes into account the operational risks of a region (for example, the risks of inflation, insolvency and/or insufficient financing of a producer, the risks of underproduction and/or overproduction).

Environmental and economic interaction between a RCC and a producer can be described according to the scheme shown in Figure 1, which reflects: 1) the financial flows contained in the matrices $A, B, C$ of the linear problem of optimal control of input actions, characterizing investment, production and financial activities of a producer, restrictions and target criteria for eco-economic interaction as an object of control; 2) the principle of managing the impact on the environment in the form of the environmental charge rate that corrects the state of an object of control and directly affects the change in the $J_{1}$ и $J_{2}$ values.

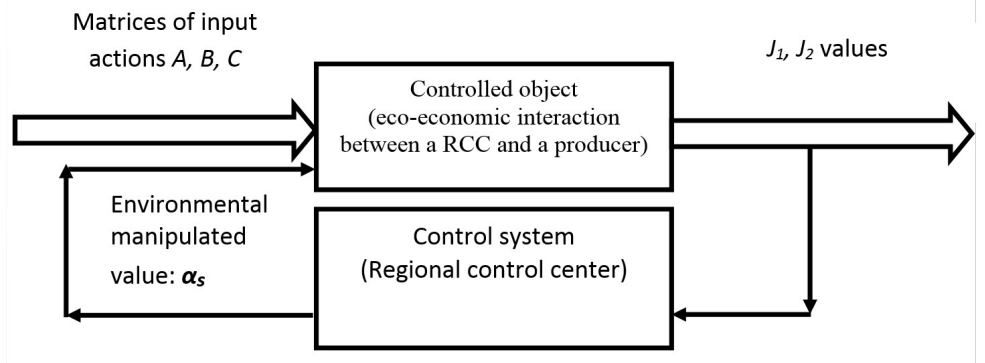

Fig. 1. General scheme for the control of the process of eco-economic interaction between a RCC and a producer.

Due to the need for the practical software implementation of the problem solution, given a large dimension and a set of its constraints, for operational support of a RCC management decision making, assume that the dependence of the $f_{i}$ functions on $\mathrm{z}$ is linear, namely $f_{i}=a_{i}$ $-b_{i} z$. The linearity of $f_{i}$ allows optimization modeling of eco-economic interaction between a RCC and a producer to create a decision support system (DSS). Assume that:

1) each type of product is produced using one set of fixed capital assets;

2) two participants of interaction - a RCC and a producer - are considered;

3) a RCC and a producer have either their own or borrowed (subsidized) funds to finance their operations (or avoid/eliminate their risks);

4) only those types of taxes and fees are taken into account, which depend on the desired investment and production volumes: value added tax (VAT), social insurance contributions (SIC), property tax (NO), income tax (IT), environmental charges and others). 


\section{Results and discussion}

Consider the following mathematical formulation of the problem of assessing ecological and economic attractiveness of a region. Let $n$ be the number of product types and, in accordance with the principle of "pure industries", sets of fixed capital assets, $x_{k}=c_{k} m_{k}-$ the optimum volume of investment in $k$-th set of fixed capital assets, where $c_{k}-$ the cost of $k$-th set, $m_{k}$ - the optimum quantity of $k$-th sets;

$x_{n+k}=P_{k} m_{k} y_{k}-$ the optimal revenue from $k$-th type product sales $(k=1, \ldots, n)$, where $P_{k}-k$-th type product unit cost, $y_{k}$ - the optimal volume of products produced using $k$-th set of fixed capital assets;

$x_{2 n+1}$ - the optimal amount of loans;

$x_{2 n+2}$ - the optimal amount of current business subsidies to a producer; $x_{2 n+2+l}(l=1, \ldots, L)-$ the optimal cost of preventing a producer's $l$-th risk, $L$ - the number of producer's risks;

$x_{2 n+2+L+m}(m=1, \ldots, M)$ - the optimal cost of preventing RCC's $m$-th risk, $M$ - the number of RCC's risks;

$a_{k}, d_{k}$ - the maximum costs incurred by a producer and a RCC, respectively, in the absence of the costs in the k-th risk direction of their performance;

$b_{k}, e_{k}$ - expert coefficients, which make sense, respectively, for each producer and RCC, assessing the significance of the effect of the costs incurred in the k-th risk direction of their performance,

$V_{k}, T_{k}$ - performance, useful life of $k$-th set of fixed capital assets;

$N_{1}=\alpha_{1} \sum_{k=1}^{n} x_{n+k}$

- annual value added tax;

$N_{2}=\alpha_{2} \sum_{k=1}^{n}\left(1-\frac{T}{T_{k}}\right) x_{k}$

- annual property tax

$N_{3}=\alpha_{3} W_{b}-$ annual income tax;

$N_{4}=\alpha_{4} \beta \sum_{k=1}^{n} x_{n+k}$

- social insurance contributions;

$N_{5}=\alpha_{5} \sum_{k=1}^{n} x_{n+k}$

- additional costs of a producer depending on the volume of production (for example, the mineral extraction tax);

$\alpha_{i}, i=1, \ldots, 5$ - respectively, the rates of value added tax, property tax, income tax, social insurance contributions and additional costs of a producer;

Shtraf $=\alpha_{S} \sum_{k=1}^{n} \frac{\xi_{k}}{P_{k}} x_{n+k} \quad$ - annual environmental charge, $\alpha_{S}$ - environmental charge rate, $\xi_{k}$ - specific pollutant emission per $k$-th type product unit; $T$ - the time bucket for the environmental and economic project; $r$ - annual discount rate of the investment project, taking into account inflation, the level of investor requirements and other economic and

political risks of producer's operations;

$$
r_{\ni}=\frac{r T}{1-(1+r)^{-T}}-1
$$
into account the dynamic characteristics of performance criteria assuming the constant profit flows and depreciation throughout the time bucket; $q_{k}$-value demand for $k$-th type products, $\delta_{k}$ - yield on capital investment (efficiency, capacity) of $k$-th set of fixed capital assets, $D_{k}$ - the maximum permissible emissions (MPE) of the pollutant from production of 
$k$-th products, $\theta_{k}$ - specific turnover costs (per unit of production) of a producer; $D S_{0}-$ proprietary initial funds; $\mathrm{Cr}$ - the optimal flow of loans to the current operations of a producer, $r_{0}$ - current business loan rate, $T_{0}$ - current business loan term, $C r_{\max }-$ the maximum possible amount of annual current business loan, Dot $t_{\max }$ - the maximum possible amount of annual subsidy to a producer, $I_{\max }$ - the maximum possible amount of producer's investment, $L_{1}, L_{2}$ - respectively, the maximum amount of the cost of avoiding (eliminating) all identified risks of a producer and a RCC.

It is easy to establish that the problem described by the mathematical model (1)-(12) has the solution, since the admissible set is a non-empty (contains a trivial solution), bounded compact. The presence of the non-trivial solution was shown using the model data be means of the author's software package.

\section{Conclusion}

Thus, the availability of the automated software package and computational experiments carried out using the mathematical model and the algorithms presented in the work allow considering them as a support system for making environmental investment decisions or, otherwise, as a toolkit for assessing a region's environmental and investment attractiveness aimed the use of innovative technologies. This makes it possible to support the adoption of eco-economic decisions by both a control center and a producer within the paradigm of sustainable development of a region, based on its system analysis, taking into account public interests and conserving the natural environment.

The article is written with the support of the Russian Foundation for Basic Research (Project No. 18410-420004 "Intellectual property as the basis for sustainable development of a coal mining region (case of Kemerovo Region)") and the Department of Education and Science of the Kemerovo Region (Agreement No. 21 of August 14, 2018).

\section{References}

1. E. N. Abanina, IOP Conf. Ser.: Earth Environ. Sci., 66, 012019 (2018)

2. T. V. Kiseleva, V. G. Mikhailov, Control Systems and Information Technologies, 48:2, 69 (2012)

3. A. D. Abalakov, N. B. Bazarova, IOP Conf. Ser.: Earth Environ. Sci., 190, 012021 (2018)

4. I. A. Rostuntsova J. Phys.: Conf. Ser., 891, 012231 (2018)

5. G.B. Dantzig, Applications of Mathematical Programming Techniques (English Universities Press, Ltd., London, 1970)

6. Susan Kinnear, Ian Ogden. A central Queensland case study Resources Policy, 39, 42 (2014)

7. S. M. Nikitenko, E.V. Goosen, IOP Conference Series: Earth and Environmental Science, 50, 012018 (2017)

8. Yu.F. Patrakov, B.A. Anferov, L.V. Kuznetsova, V.I. Klishin, S.M. Nikitenko, XVIII International Coal Preparation Congress, 493 (2016)

9. P.D. Kosinskiy, V.V. Merkur'ev, A.V. Medvedev, The 8-th Russian-Chinese Symposium Coal in the 21-st Century: Mining, Processing and Safety, 1, 366 (2016)

10. L. Ke, L. Boqiang. Energy \& Environment, 26, 1015 (2015) 